\title{
Comparison of an Optical and a Mechanical Navigation System
}

\author{
S. Martelli, S. Bignozzi, M. Bontempi, S. Zaffagnini, and L. Garcia \\ Laboratorio di Biomeccanica, Istituti Ortopedici Rizzoli, Bologna, Italy \\ \{s.martelli, s.bignozzi, m.bontempi, \\ s.zaffagnini\}@biomec.ior.it \\ http://www.ior.it/biomec/
}

\begin{abstract}
Different technologies have been used for computer assisted orthopaedic surgery for acquisition of surfaces and motions, but the most efficient are optical and mechanical[2]. In this study we compared two specific systems of these categories: FlashPoint ${ }^{\mathrm{TM}} 5000$ (Image Guided Technologies, USA) and FAROArm ${ }^{\text {TM }}$ (FARO Technologies, USA). FARO Arm showed an intrinsic uncertainty of $0.1 \mathrm{~mm}$ overcoming FlashPoint $(0.2 \mathrm{~mm})$. However their use in noisy conditions resulted in similar application-uncertainty. FARO Arm can track motion at $31 \mathrm{~Hz}$ while FlashPoint at $17 \mathrm{~Hz}$, therefore FlashPoint spatial resolution may be critical during fast movements. In simulated medical applications FARO Arm showed some difficulties (i) when used for passive motion with negligible forces, because the surgeon has to compensate its weight and (ii) during sample trajectory, because it has isolated singularities within its workspace. FlashPoint was affected by reflected light and required complete visibility during tracking of large trajectories. However its use in in-vivo conditions is more promising than FARO-Arm's.
\end{abstract}

\section{Introduction}

Computer technologies have increased the knowledge in orthopedic surgery by numerically describing or quantifying anatomical and cinematic data and represent an intriguing perspective for the future. Several systems can be used in computer assisted orthopaedic surgery [2]. So far the most commonly used and efficient are optical and mechanical systems [7].

The aim of this study was to compare two specific systems of these categories: FlashPoint ${ }^{\mathrm{TM}} 5000$ (Image Guided Technologies Inc., Boulder, Colorado, USA) and FARO ${ }^{\mathrm{TM}}$ Arm (FARO Technologies, Lake Mary, Florida, USA). We choose these two systems as representative of their category, despite some differences in devices of the same kind [3],[5], to compare two different approaches in computerassisted orthopaedic surgery. We evaluated the efficacy of both systems and the possible influence of environmental conditions, as well as the performance for typical tasks of computer assisted and navigated surgery. This study complements the few previous works on trackers' or navigators' evaluation, which analyze either optical devices or single applications [3],[5],[7]. 


\section{Examined Systems}

\subsection{FLASHPOINT}

FlashPoint is an optical 3D localizer which utilizes infrared technology (Figure 1). It consists of an assembly of three high resolution cameras, able to localize infrared emitters, and of a control unit with an internal microprocessor and ROM storing FlashPoint software. Cameras measure the coordinate location of the emitters (sterilizable with ethylene oxide gas) placed on instruments and send them to the unit control. Data acquisition can be controlled in two different ways, via software or using the button probe connected to the breakout box data flow can also be settled as "continuous" of "single point" mode.

In our experiments we relied on the manufacturer's software to identify the probe location and we elaborated 3D data off-line mainly for 3D display, with custom software, accuracy tests or specific medical application, anatomical and kinematic elaborations. In medical applications we used FlashPoint both as localizer and as tracker, testing its performances and usability.

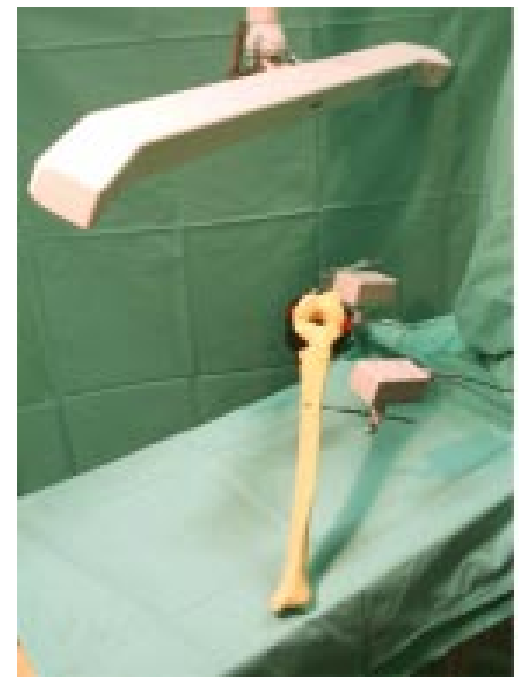

Fig. 1. FlashPoint set-up

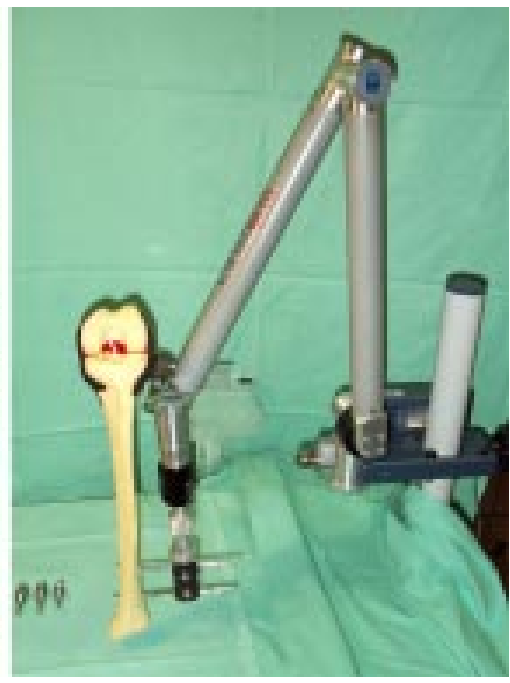

Fig. 2. FARO Arm set-up

\subsection{FARO Arm}

FARO Arm is a mechanical 3D passive electrogoniometer with anthropomorphic structure. We used the model B0601/02m which has its 6-degree-of-freedom (DOF) distributed in a human arm-like configuration, that is 3 concurrent and mutually perpendicular revolute links at the "wrist", 1 at the "elbow" and 2 at the "shoulder" (Figure 2).

FARO Arm weights $4 \mathrm{~kg}$ and is mounted on a heavy and stable clamping device which makes the sensor easy to move and handle for digitalization. FARO Arm can 
be used in the operating theatre covered with sterile draping and all end-effectors can be sterilized in autoclave. FARO Arm acquires the position and orientation of its endeffectors through a custom control unit, either stream mode or point mode.

In our experiments we relied on the manufacturer's software to identify the probe location and we elaborated 3D data off-line mainly for 3D display, accuracy tests or specific medical application. In medical applications we used FARO Arm both as localizer and as tracker, testing its performances and usability.

\section{Experimental Evaluation of the Systems - Methods}

\subsection{Digitizing Performances}

\section{Single Point Acquisition and Noise Sensitivity}

The uncertainty of a single point acquisition was tested acquiring 9 points located on a plane through the origin of the sensors' workspace with XY and YZ orientations. The 9 points were distributed on a plane positioned $(150.0 \pm 0.1) \mathrm{cm}$ under FlashPoint central camera at increasing distance from its centre, in order to test sub-volumes in its workspace and the central $150 \mathrm{~cm}$ of FARO Arm's spherical workspace. Each point was acquired 200 times in stream mode using the single LED digitiser with FlashPoint and the point probe in different orientations with FARO Arm. We defined the uncertainty on the acquired point as the sizes of the parallelepiped containing the $75 \%$ of the points. To simulate the possible noise in FlashPoint workspace we used 2 lamps with different spectrum (L) directed to the probe and a mirror (M) reflecting the emitters' light towards cameras. To analyse the optical properties of FlashPoint, we repeated the single point acquisition test in four different conditions: complete darkness (-L-M), environmental light (+L-M), darkness plus noise $(-\mathrm{L}+\mathrm{M})$, environmental light plus noise $(+\mathrm{L}+\mathrm{M})$. To simulate the possible noise in FARO Arm workspace we repeated the single point acquisition test with FARO Arm in a temperature controlled room, keeping the sensor in the room an hour before the measurements. We tested FARO Arm performances at $10^{\circ} \mathrm{C}, 20^{\circ} \mathrm{C}, 30^{\circ} \mathrm{C}, 40^{\circ} \mathrm{C}$.

\section{Distances and Angles Acquisition}

From the data obtained on single point acquisition we calculated the uncertainty on the distances $(d)$ between two points $\mathrm{P}_{1}=\left(\mathrm{x}_{1}, \mathrm{y}_{1}, \mathrm{z}_{1}\right), \mathrm{P}_{2}=\left(\mathrm{x}_{2}, \mathrm{y}_{2}, \mathrm{z}_{2}\right)$ using the standard propagation error theory [6]. In particular the following formula holds

$$
\Delta d=\frac{\sqrt{\left(x_{2}-x_{1}\right)^{2}\left(x_{1}^{2} \Delta x_{1}^{2}+x_{2}^{2} \Delta x_{2}^{2}\right)+\left(y_{2}-y_{1}\right)^{2}\left(y_{1}^{2} \Delta y_{1}^{2}+y_{2}^{2} \Delta y_{2}^{2}\right)+\left(z_{2}-z_{1}\right)^{2}\left(z_{1}^{2} \Delta z_{1}^{2}+z_{2}^{2} \Delta z_{2}^{2}\right)}}{\sqrt{\left(x_{2}-x_{1}\right)^{2}+\left(y_{2}-y_{1}\right)^{2}+\left(z_{2}-z_{1}\right)^{2}}}
$$

where $\Delta \mathrm{d}$ is the uncertainty on the measured distances, and $\Delta \mathrm{x}, \Delta \mathrm{y}, \Delta \mathrm{z}$ are the acquisition errors on single points coordinates.

Similarly the uncertainty on angles were obtained considering three points $\mathrm{P}_{1}=\left(\mathrm{x}_{1}, \mathrm{y}_{1}\right.$, $\left.\mathrm{z}_{1}\right), \mathrm{P}_{2}=\left(\mathrm{x}_{2}, \mathrm{y}_{2}, \mathrm{z}_{2}\right), \mathrm{P}_{3}=\left(\mathrm{x}_{3}, \mathrm{y}_{3}, \mathrm{z}_{3}\right)$, two vectors obtained by subtraction from the 3 
points with origin in $\mathrm{P}_{2}\left(\mathrm{v} 1=\left(\mathrm{x}_{1}-\mathrm{x}_{2}, \mathrm{y}_{1}-\mathrm{y}_{2}, \mathrm{z}_{1}-\mathrm{z}_{2}\right), \mathrm{v}_{2}=\left(\mathrm{x}_{3}-\mathrm{x}_{2}, \mathrm{y}_{3}-\mathrm{y}_{2}, \mathrm{z}_{3}-\mathrm{z}_{2}\right)\right)$ and the cosines of the angle $(\theta)$ between $\mathrm{v}_{1}$ and $\mathrm{v}_{2}$. The following formula holds

$$
\Delta \cos (\theta)=\sqrt{\sum_{i=x, y, z}\left[\left(\frac{\partial \cos (\theta)}{\partial i_{1}} \Delta i_{1}\right)^{2}+\left(\frac{\partial \cos (\theta)}{\partial i_{2}} \Delta i_{2}\right)^{2}+\left(\frac{\partial \cos (\theta)}{\partial i_{3}} \Delta i_{3}\right)^{2}\right]}
$$

where $\Delta \cos (\theta)$ is the uncertainty on the measured angle, and $\Delta x_{1,2,3}, \Delta y_{1,2,3}, \Delta z_{1,2,3}$ are the acquisition uncertainty on single points coordinates.

\section{$3 D$ Solid Reconstruction}

To verify accuracy of $3 \mathrm{D}$ reconstructions we acquired three solids with basic geometric shape: 1) a flat steel surface, with a tolerance of $0.01 \mathrm{~mm}$; 2) a Perspex $70 \mathrm{~mm} \times 48 \mathrm{~mm} \times 300 \mathrm{~mm}( \pm 0.05 \mathrm{~mm})$ parallelepiped specimen; 3) a Perspex cylinder with a height equal to $200 \mathrm{~mm}$, circular section of radius $35 \mathrm{~mm}$ and tolerance $0.05 \mathrm{~mm}$.

They were acquired by the two sensors with a spatial resolution of $1 \mathrm{~mm}$. The cloud of $3 \mathrm{D}$ points thus obtained was displayed and the dimensions and shape of the objects were evaluated in 2D suitable sections and least square interpolation of the 3D points. An experimental acquisition of a foam knee was used to interview the surgeon about the sensors' usability during digitisation[4].

\subsection{Tracking Performances}

\section{Temporal Sampling Rate}

The intrinsic temporal sampling rate (TSR, $\omega_{i}$ ) was measured counting the number of acquisitions (n) of a fixed point during a time interval $t=(20 \pm 1 \mathrm{sec})$, that is:

$$
\omega_{i}=\frac{n}{t}
$$

Therefore the associated uncertainty $\left(\Delta \omega_{i}\right)$ is $[1]$ :

$$
\Delta \omega_{i}=\frac{\Delta t}{t} \omega_{i}
$$

With FlashPoint the acquisition was tested in the following modalities: via keyboard control or using the button connected to the computer. We repeated all the acquisitions with and without the confirmation sound, in order to evaluate its effect on performances. With FARO Arm continuous acquisition can be done only in one modality.

\section{Spatial Sampling Distance}

In both systems during stream mode acquisition of a surface, path or trajectory (that we will call "dynamic"), the spatial sampling distance SSD, that is minimal distance between two consecutive points, is settable via software. 
The purpose of our measurements was to verify the relationship between the SSD and the temporal sampling rate in these conditions $\left(\omega_{d}\right)$. As the intrinsic temporal sampling rates of the devices are fixed (compare with \$3.2), the SSD $(d)$, the TSR $\left(\omega_{d}\right)$ and the velocity of the probe during dynamic acquisition are linked by the following formula:

$$
v=\omega_{d} \cdot d \quad \forall v, d \quad \omega_{d} \leq \omega_{i}
$$

Therefore we expected to have a reliable spatial sampling in stream mode only when the probe velocity is less than a so-called "critical velocity" $\left(v_{c}\right)$

$$
v_{c}=\omega_{i} \cdot d
$$

Our experiment consisted in 10 repeated acquisitions in a linear trajectory. SSD was fixed $(d=2 \mathrm{~mm})$; the probe was moved at different velocities positioning the sensors on a trolley free to move on a rectilinear track attached to a falling weight; the dynamic TSR was computed from (7).

\section{D Trajectory Reconstruction}

The accuracy of the dynamic computations was evaluated measuring the relative motion of two sample objects during translations and rotations in the sensors' workspace.

In a first experiment we tracked the translation of the Perspex parallelepiped on the flat surface, along two perpendicular directions, measuring the linearity of the recorded trajectory, the performed distance and the position of the recorded lines with respect to the sensor. We performed translation in two perpendicular directions of the sensors' workspaces moving by $10,20,40,60 \mathrm{~cm}$.

In a second experiment we tracked the translation and the rolling motion of the Perspex cylinder on the flat surface, along two perpendicular directions. Also in this case we measured the shape and the size of the recorded trajectories as acquired by both sensors. We performed rotations around 2 perpendicular axes (parallel to the flat surface) moving by $10^{\circ}, 20^{\circ}, 30^{\circ}, 60^{\circ}$.

An experimental acquisition of flexion-extension motion of a foam knee was used to interview the surgeon about the sensors' performances during dynamic tracking.

\section{Experimental Evaluation of the Systems - Results}

\subsection{Digitizing Performances}

\section{Single Point Acquisition and Noise Sensitivity}

The following tables report the mean uncertainty on the 9 points acquired during the single point acquisitions with the two sensors under different environment conditions. 
Table 1. Single point uncertainty for FlashPoint under different light conditions.

\begin{tabular}{cccc}
\hline Temperature $\left({ }^{\mathbf{0}} \mathbf{C}\right)$ & $\mathbf{X}$ & Uncertainty $(\mathbf{m m})$ along \\
& 0.02 & $\mathbf{Y}$ & $\mathbf{X}$ \\
\hline -L-M & 0.02 & 0.02 & 0.05 \\
+L-M & 0.04 & 0.02 & 0.06 \\
-L+M & 0.04 & 0.04 & 0.08 \\
+L+M & 0.04 & 0.09 \\
\hline
\end{tabular}

Table 2. Single point uncertainty for FARO Arm at different temperatures.

\begin{tabular}{cccc}
\hline Temperature $\left({ }^{\mathbf{0}} \mathbf{C}\right)$ & $\mathbf{X}$ & Uncertainty $(\mathbf{m m})$ along & $\mathbf{X}$ \\
& $\mathbf{X}$ & $\mathbf{Y}$ & 0.05 \\
$\mathbf{1 0} \pm \mathbf{1}$ & 0.05 & 0.05 & 0.05 \\
$\mathbf{2 0} \pm \mathbf{1}$ & 0.05 & 0.05 & 0.05 \\
$\mathbf{3 0} \pm \mathbf{1}$ & 0.05 & 0.05 & 0.05 \\
$\mathbf{4 0} \pm \mathbf{1}$ & 0.05 & 0.05 & \\
\hline
\end{tabular}

Distances and Angles Acquisition

FlashPoint has an uncertainty of $0.2 \mathrm{~mm} / 0.2^{\circ}$ in distance and angle computation, FARO Arm an uncertainty of $0.1 \mathrm{~mm} / 0.1^{\circ}$.

\section{Solid Reconstruction}

Surface data acquired with FlashPoint and FARO Arm were similar when acquired in stream or point mode. The results of experimental reconstructions of geometric models is reported in Table 3.

Table 3. Uncertainty of $3 \mathrm{D}$ reconstruction

\begin{tabular}{|c|c|c|c|}
\hline & & FlashPoint & Faro \\
\hline \multirow{3}{*}{$\begin{array}{l}\text { Parallelepiped sizes } \\
\text { (mm) }\end{array}$} & Height & $300.2 \pm 0.2$ & $299.9 \pm 0.2$ \\
\hline & Width & $70.1 \pm 0.2$ & $70.9 \pm 0.2$ \\
\hline & Thickness & $48.1 \pm 0.2$ & $48.1 \pm 0.1$ \\
\hline \multirow[t]{2}{*}{ Cylinder size } & Height & $200.0 \pm 0.2$ & $200.0 \pm 0.1$ \\
\hline & Radius & $34.1 \pm 0.2$ & $35.0 \pm 0.1$ \\
\hline Residual on flat surface fitting & $(\mathrm{mm})$ & $<0.1$ & $<0.1$ \\
\hline
\end{tabular}


The surgeon could not find any difference between FlashPoint and FARO Arm performances while digitizing the knee model and could always find visible orientations of the Flash Point probe or reachable configurations of FARO Arm endeffector to digitize knee surfaces. Stream mode was used as often as possible with both sensors.

\subsection{Tracking Performances}

\section{Temporal Sampling Rate}

FlashPoint showed an experimental TSR $\omega_{i}=(17.32 \pm 0.07) \mathrm{Hz}$ without confirmation sound and $\omega_{i}=(9.28 \pm 0.05) \mathrm{Hz}$ with confirmation sound for all acquisition modality.

FARO Arm showed an experimental TSR $\omega_{i}=(31.4 \pm 0.5) \mathrm{Hz}$

\section{Spatial Sampling Distance}

With a SSD of $2 \mathrm{~mm}$ the computed critical velocity of FlashPoint is $34 \mathrm{~mm} / \mathrm{s}$, for FARO Arm is $63 \mathrm{~mm} / \mathrm{s}$. In our experiments respected SSD set by the user, when moving below the critical velocity. In this case the TSR can be computed as $\omega_{d}=v / d$, in particular FARO Arm rarely reached its critical velocity during kinematics' tests. On the contrary over the critical velocity of the probe the achieved SSR depends on the probes velocity and is obtained as $d=v / \omega_{i}$.

\section{Trajectories Reconstruction}

For both sensors there were no differences in measured translations between the parallelepiped and the cylinder.

With FlashPoint 3D distances between first and last position of the recorded trajectories were correct within $0.30 \mathrm{~mm}$ of error for all motions, with FARO Arm within $0.27 \mathrm{~mm}$. The linear trajectory was fitted by a line with a residual $<0.1 \mathrm{~mm}$ for both sensors and all acquisitions. The amount of rotation performed experimentally could be measured experimentally with $0.5^{\circ}$ of error for FlashPoint and FARO Arm in the two tested directions and all amounts.

The surgeon found easier the execution of flexion-extension with FlashPoint then with FARO Arm, as he felt that the latter sensor produced a bigger constraint to be compensated in quasi-unconstrained joint configuration. Moreover the adjustment of the joint position with respect to FlashPoint took tests to adjust visibility during the whole motion, against four tests to avoid FARO Arm's singularities during the flexion. 


\section{Discussion}

FlashPoint and FARO Arm showed constant submillimetric accuracy in the whole workspace. The different technical features of the two sensors result in different workspaces $(100 \mathrm{~cm}$ spherical workspace for FlashPoint versus $180 \mathrm{~cm}$ spherical workspace for FARO Arm) and different experimental uncertainties $(0.2 \mathrm{~mm}$ for FlashPoint versus $0.1 \mathrm{~mm}$ for FARO Arm). However the capacity of both sensors to reproduce 3D geometry within $0.2 \mathrm{~mm}$ uncertainty is sufficient in medical applications to improve the results of any computer-assisted procedure with respect to manual ones.

In our tests we have also measured different intrinsic temporal sampling rates $(17 \mathrm{~Hz}$ for FlashPoint versus $31 \mathrm{~Hz}$ for FARO Arm), which affect the spatial resolutions during dynamic acquisitions. However in medical applications tracking is used for relatively slow motion (such as manual diagnostic tests) which can be easily tracked by both sensors. The most important feature in dynamic tests is the sensor versatility and usability and we can notice that the FARO Arm weight could be critical in some passive motions and the singularities in its workspace obliges to careful tests of the initial set-up.

In conclusion we can remark that FlashPoint can be significantly affected by lights artifacts, such as special surgical tools or lamps, but when used in open field with no occlusions it is not influenced by the structure under examination. Due to the possibility of FlashPoint of tracking more than one probe simultaneously and its ease of use it is very natural for in vivo applications, while FARO Arm requires a firmly fixation of the reference anatomical part and therefore is more affected by environmental vibrations and is more invasive. On the other side FARO Arm can be used in smaller spaces with respect to FlashPoint.

\section{References}

1. Capolaroni M. et al.: La misura e la valutazione della sua incertezza nella fisica sperimentale. Zanichelli 1987

2. Chassat F. et al.: Experimental protocol of accuracy evaluation of 6-D localizers for computer-Integrated Surgery: Application to four optical localizers. Miccai Proceedings, 1998

3. Khadem R. et al.: Comparative tracking error analysis of five different Optical tracking systems, Computer Aided Surgery. 2000 (5): 97-107

4. Martelli S.: A new method for simultaneous anatomical and functional studies of articular and its application to the human knee", Computer methods and programs in biomedicine, 2003 (70): 223-240

5. Qinghang Li et al.: Effect of optical digitizer selection on the application accuracy of a surgical localization system-A quantitative comparison between the Optotrack and FlashPoint tracking systems. Computer Aided Surgery, 1999 (4): 314-32

6. Taylor J.R.: An introduction to error analysis, the study of uncertainties in physical measurements. University Science Books 1982

7. Troccaz J. et al.: Guiding systems for computer-assisted surgery: introducing synergistic devices and discussing different approaches. Medical Image Analysis, 1998 (2): 101-119 